PROCEEDINGS OF THE

AMERICAN MATHEMATICAL SOCIETY

Volume 128, Number 3, Pages 873-876

$\mathrm{S}$ 0002-9939(99)05267-3

Article electronically published on July 6, 1999

\title{
LIPSCHITZ CONTINUITY OF OBLIQUE PROJECTIONS
}

\author{
HARALD K. WIMMER
}

(Communicated by David R. Larson)

\begin{abstract}
Let $W$ and $L$ be complementary spaces of a finite dimensional unitary space $V$ and let $P(W, L)$ denote the projection of $V$ on $W$ parallel to $L$. Estimates for the norm of $P(W, L)-P(W, M)$ are derived which involve the norm of the restriction of $P(W, L)$ to $M$ or the gap between $L$ and $M$.
\end{abstract}

\section{INTRODUCTION AND PRELIMINARIES}

Let $V=W \oplus L$ be a nontrivial direct sum decomposition of an $n$-dimensional unitary space $V$ and let $P(W, L)$ denote the oblique projection on $W$ along $L$. If the distance between subspaces is measured in the gap metric, then all subspaces $M$ contained in a sufficiently small neighbourhood $U(L)$ of $L$ are also complementary to $W$ (see e.g. [1, p. 390] or [5]). For $M \in U(L)$ set $\pi(M)=P(W, M)$. In this note we study the map $\pi(M)$. An estimate for $\|\pi(M)-\pi(L)\|$ will be obtained which involves the restriction of $P(W, L)$ to $M$. A Lipschitz constant for $\pi$ in [1] will be improved.

Notation. For a linear map $A: Y \rightarrow V$ the norm $\|A\|$ denotes the operator norm, i.e. $\|A\|=\sup \{\|A y\|, y \in Y,\|y\|=1\}$. Let $P_{W}$ denote the orthogonal projection of $V$ on $W$ and set

$$
P(W, L ; M)=\left.P(W, L)\right|_{M} .
$$

We write $d(x, M)$ for the distance of $x \in V$ from $M$. The gap between two subspaces $L$ and $M$ is defined by

$$
\theta(L, M)=\left\|P_{L}-P_{M}\right\| .
$$

We shall need the following facts on the gap, for which we refer to [2] and [1]. First of all $\theta$ is a metric on the set of subspaces of $V$, and $\theta(L, M) \leq 1$. If $\theta(L, M)<1$, then

$$
\operatorname{dim} M=\operatorname{dim} L .
$$

In the case of (1.1) we have

$$
\theta(L, M)=\left\|P_{L}\left(I-P_{M}\right)\right\|=\left\|P_{M}\left(I-P_{L}\right)\right\| .
$$

Received by the editors August 10, 1997 and, in revised form, April 28, 1998. 1991 Mathematics Subject Classification. Primary 51M05, 51M16, 15 A45.

Key words and phrases. Oblique projections, direct complements.

(C)1999 American Mathematical Society 
Lemma 1.1. Assume $V=W \oplus L$.

(i) For a subspace $M$ of $V$ we have

$$
\|P(W, L ; M)\|=\left\|P(W, L) P_{M}\right\| .
$$

(ii) If $\operatorname{dim} M=\operatorname{dim} L$, then

$$
\|P(W, L ; M)\| \leq\|P(W, L)\| \theta(L, M) .
$$

Proof. (i) For $y \in M, M \neq 0$, we have $P(W, L ; M) y=P(W, L) P_{M} y$. Therefore

$$
\begin{aligned}
& \|P(W, L ; M)\|=\max \left\{\frac{\left\|P(W, L) P_{M} y\right\|}{\|y\|}, y \neq 0, y \in M\right\} \leq\left\|P(W, L) P_{M}\right\| \\
& =\max \left\{\frac{\left\|P(W, L) P_{M} y\right\|}{\|y\|}, y \neq 0, y \in V\right\} \\
& \leq \max \left\{\frac{\left\|P(W, L) P_{M} y\right\|}{\left\|P_{M} y\right\|}, y \in V, P_{M} y \neq 0\right\}=\|P(W, L ; M)\| .
\end{aligned}
$$

(ii) From $P(W, L)=P(W, L)\left(I-P_{L}\right)$ and (1.3) follows

$$
\|P(W, L ; M)\|=\left\|P(W, L)\left(I-P_{L}\right) P_{M}\right\| .
$$

Hence (1.2) yields (1.4).

The following observations do not seem to be widely known.

Lemma 1.2. Assume $V=W \oplus L$.

(i) If $W \neq 0$, then

$$
\max _{x \in W,\|x\|=1} \frac{1}{d(x, L)}=\|P(W, L)\| .
$$

(ii) If $W \neq 0$ and $L \neq 0$, then

$$
\|P(W, L)\|=\|P(L, W)\| .
$$

Proof. (i) We shall see that (1.5) is equivalent to the identity

$$
\frac{1}{1-\left\|P_{L} P_{W}\right\|^{2}}=\|P(W, L)\|^{2},
$$

which is due to Ljance [3] (see [4] or [6]). Set

$$
\tau=\min _{x \in W,\|x\|=1} d(x, L)=\min _{x \in W,\|x\|=1}\left\|\left(I-P_{L}\right) P_{W} x\right\| .
$$

Then the left-hand side of (1.5) is equal to $1 / \tau$. If $x \in W$ and $\|x\|=1$, then

$$
\left\|\left(I-P_{L}\right) P_{W} x\right\|^{2}+\left\|P_{L} P_{W} x\right\|^{2}=1 .
$$

Hence

$$
\tau^{2}=1-\max _{x \in W,\|x\|=1}\left\|P_{L} P_{W} x\right\|^{2}=1-\left\|P_{L} P_{W}\right\|^{2} .
$$

Therefore

$$
\frac{1}{\tau^{2}}=\frac{1}{1-\left\|P_{L} P_{W}\right\|^{2}},
$$

and (1.5) follows from (1.7).

(ii) Since $P_{L} P_{W}=0$ implies $P_{L} P_{W} P_{L}=0$ and thus $P_{W} P_{L}=0$, we note that either $P_{L} P_{W}=P_{W} P_{L}=0$ or both $P_{L} P_{W} \neq 0$ and $P_{W} P_{L} \neq 0$. In each case we have $\left\|P_{L} P_{W}\right\|=\left\|P_{W} P_{L}\right\|$. Hence (1.7) implies (1.6). 


\section{Estimates FOR OBLIQUE PROJECTIONS}

Theorem 2.1. Assume $V=W \oplus L, W \neq 0, L \neq 0$.

(i) Let $M$ be a subspace of $V$ with $\operatorname{dim} M=\operatorname{dim} L$ and

$$
\mu=\|P(W, L ; M)\|<1 \text {. }
$$

Then

$$
V=W \oplus M
$$

and

$$
\|P(W, M)-P(W, L)\| \leq \frac{\mu}{1-\mu}\|P(W, L)\| .
$$

(ii) If a subspace $M$ satisfies

$$
\theta(L, M) \leq(1-c)\|P(W, L)\|^{-1}, \quad 0<c<1,
$$

then we have (2.2) and

$$
\|P(W, M)-P(W, L)\| \leq \frac{1}{c}\|P(W, L)\|^{2} \theta(L, M) .
$$

Proof. (i) Suppose $x \neq 0$ for some $x \in W \cap M$. Then $P(W, L ; M) x=x$. Hence $\|P(W, L ; M)\| \geq 1$, which contradicts (2.1). Therefore we have $W \cap M=0$ and (2.2). Now put $S=P(M, W) P_{L}$. Then

$$
P(M, W)\left[I-P_{L} P(L, W)\right]=P(M, W) P(W, L)=0
$$

implies $P(M, W)=S P(L, W)$. Using $P(W, L)=I-P(L, W)$ and (1.6) we obtain

$$
\begin{aligned}
& \|P(W, M)-P(W, L)\|=\|P(M, W)-P(L, W)\| \\
& =\left\|S P(L, W)-P_{L} P(L, W)\right\| \leq\left\|S-P_{L}\right\|\|P(W, L)\| .
\end{aligned}
$$

Thus our target inequality is

$$
\left\|S-P_{L}\right\| \leq \frac{\mu}{1-\mu} .
$$

Since $P(L, W) P(W, M) x=0$ for all $x \in V$, we have $P(L, W)[I-P(M, W)]=0$ or $P(L, W) P(M, W)=P(L, W)$. Similarly $P(L, W) P_{L}=P_{L}$. Hence

$$
P(L, W) P(M, W) P_{L}=P(L, W) S=P_{L},
$$

and we obtain $S-P_{L}=[I-P(L, W)] S=P(W, L) P_{M} S$. Then

$$
\begin{aligned}
\left(S-P_{L}\right)^{*}\left(S-P_{L}\right) & =S^{*} S-P_{L} S-S^{*} P_{L}+P_{L} \\
& =S^{*} P_{M} P(W, L)^{*} P(W, L) P_{M} S,
\end{aligned}
$$

which implies

$$
P_{L} S+S^{*} P_{L}=S^{*}\left[I-P_{M} P(W, L)^{*} P(W, L) P_{M}\right] S+P_{L} .
$$

For the left-hand side of (2.8) we obtain

$$
\left\|P_{L} S+S^{*} P_{L}\right\| \leq 2\left\|P_{L} S\right\| \leq 2\left\|P_{L}\right\|\|S\| \leq 2\|S\| .
$$

Put $T=I-P_{M} P(W, L)^{*} P(W, L) P_{M}$ such that the right-hand side of (2.8) equals $R=S^{*} T S+P_{L}$. Since $P_{L}$ is the identity map on $L$ and $S P_{L}=S$, it is not difficult to show that $\|R\|=\left\|S^{*} T S\right\|+1$. Now (2.1) implies that $T$ is positive definite and that $1-\left\|P(W, L) P_{M}\right\|=1-\mu^{2}>0$ is the smallest eigenvalue of $T$. Hence $\left\|S^{*} T S\right\| \geq\|S\|^{2}\left(1-\mu^{2}\right)$. Thus $\|S\|$ satisfies

$$
0 \leq\|S\|^{2}\left(1-\mu^{2}\right)+1 \leq 2\|S\|,
$$


which is equivalent to

$$
0<\frac{1}{1+\mu} \leq\|S\| \leq \frac{1}{1-\mu} .
$$

Then (2.7) yields

$$
\left\|S-P_{L}\right\| \leq\|S\|\left\|P(W, L) P_{M}\right\| \leq \frac{\mu}{1-\mu},
$$

and we have (2.6), which completes the proof of (2.3).

(ii) From (2.4) and (1.4) we obtain

$$
\mu \leq\|P(W, L)\| \theta(L, M) \leq 1-c<1 .
$$

Then $\|P(W, L)\| \geq 1$ implies $\theta(L, M)<1$ and $\operatorname{dim} M=\operatorname{dim} L$. Because of $\mu<1$ we can use (i) and conclude that $P(W, M)$ exists. Since $0 \leq \mu \leq 1-c$ is equivalent to

$$
0 \leq \frac{1}{1-\mu} \leq \frac{1}{c}
$$

the estimate (2.5) follows immediately from (2.3).

In the neighbourhood of $L$ given by (2.4) the estimate (2.5) yields a Lipschitz constant for $P(W, M)$ of the form

$$
\frac{1}{c}\|P(W, L)\|^{2} .
$$

In $[1$, p. 390] we find for sufficiently small $\theta(L, M)$ an estimate

$$
\|P(W, M)-P(W, L)\| \leq K \theta(L, M)
$$

with

$$
K=2\|P(W, L)\| \max _{x \in W,\|x\|=1} \frac{1}{d(x, L)} .
$$

According to Lemma 1.2 the Lipschitz constant $K$ in (2.12) is equal to (2.11) with $c=1 / 2$.

\section{REFERENCES}

[1] I. Gohberg, P. Lancaster, and L. Rodman, Invariant Subspaces of Matrices with Applications. Wiley, New York, 1986. MR 88a:15001

[2] T. Kato, Perturbation Theory for Linear Operators. Springer, Berlin, 1996. MR 96a:47025

[3] V.E. Ljance, Some properties of idempotent operators. Teor. i. Prikl. Mat. L'vov, 1:16-22, 1959 (in Russian).

[4] V. Pták, Extremal operators and oblique projections. C̆asopis Pĕst. Mat., 110:343-350, 1985. MR 87g:47053

[5] J.M. Schumacher. A pointwise criterion for controller robustness. Systems Control Lett., 18:18, 1992. MR 93b:93091

[6] H.K. Wimmer, Canonical angles of unitary spaces and perturbations of direct complements. Linear Algebra Appl., 287:373-379, 1999.

Mathematisches Institut, Universität Würzburg, D-97074 Würzburg, Germany

E-mail address: wimmer@mathematik.uni-wuerzburg.de 